\title{
Review Article \\ Brain-Derived Neurotrophic Factor Polymorphism and Aphasia after Stroke
}

\author{
Nathan T. Lee, ${ }^{1}$ Fatimah Ahmedy $\mathbb{D}^{1}{ }^{1}$ Natiara Mohamad Hashim $\mathbb{D}^{2},{ }^{2}$ Khin Nyein Yin $\left(\mathbb{D},{ }^{3}\right.$ \\ and Kai Ling Chin $\mathbb{1}^{4}$ \\ ${ }^{1}$ Rehabilitation Medicine Unit, Faculty of Medicine \& Health Sciences, Universiti Malaysia Sabah, Kota Kinabalu, Malaysia \\ ${ }^{2}$ Department of Rehabilitation Medicine, Faculty of Medicine, Universiti Teknologi MARA, Sg. Buloh, Malaysia \\ ${ }^{3}$ Department of Surgery, Faculty of Medicine \& Health Sciences, Universiti Malaysia Sabah, Kota Kinabalu, Malaysia \\ ${ }^{4}$ Department of Biomedical Sciences, Faculty of Medicine \& Health Sciences, Universiti Malaysia Sabah, Kota Kinabalu, Malaysia
}

Correspondence should be addressed to Fatimah Ahmedy; fatimahmedy@ums.edu.my

Received 15 September 2020; Accepted 11 July 2021; Published 28 July 2021

Academic Editor: Elisa Rubino

Copyright $\odot 2021$ Nathan T. Lee et al. This is an open access article distributed under the Creative Commons Attribution License, which permits unrestricted use, distribution, and reproduction in any medium, provided the original work is properly cited.

Stroke is one of the most deliberating causes of mortality and disability worldwide. Studies have implicated Val66Met polymorphism of the brain-derived neurotrophic factor (BDNF) gene as a genetic factor influencing stroke recovery. Still, the role of BDNF polymorphism in poststroke aphasia is relatively unclear. This review assesses the recent evidence on the association between the BDNF polymorphism and aphasia recovery in poststroke patients. The article highlights BNDF polymorphism characteristics, speech and language interventions delivered, and the influence of BNDF polymorphism on poststroke aphasia recovery. We conducted a literature search through PubMed and Google Scholar with the following terms: "brain derived-neurotrophic factor" and "aphasia" for original articles from January 2000 until June 2020. Out of 69 search results, a detailed selection process produced a total of 3 articles that met the eligibility criteria. All three studies included Val66Met polymorphism as the studied human BDNF gene. One of the studies demonstrated insufficient evidence to conclude that BDNF polymorphism plays a role in poststroke aphasia recovery. The remaining two studies have shown that Met allele genotype (either single or double nucleotides) was associated with poor aphasia recovery, in either acute or chronic stroke. Carriers of the Val66Met polymorphism of BDNF gave a poorer response to aphasia intervention and presented with more severe aphasia.

\section{Introduction}

Stroke is one of the leading causes of death and acquired disability globally [1]. In addition, many demographic and clinical factors have influenced poststroke recovery, including age, stroke severity, presence of cognitive impairment, and neuropsychological deficits [2]. Thus, there is an emerging interest in studying genetic factors and variations that influence stroke susceptibility and recovery [3]. One genetic variation of interest is the Val66Met single-nucleotide polymorphism of the brain-derived neurotrophic factor (BDNF) gene in humans, a potential clinically significant genetic variation associated with stroke risk and prognosis [3]. The BDNF Val66Met polymorphism structurally involves the substitution of the amino acid valine ( $\mathrm{Val}$ ), to methionine (Met), in the $5^{\prime}$ ori-region of the human BDNF gene [4].

BDNF, being part of the neurotrophin family of growth factors, is believed to influence a wide range of aspects of the nervous system, including but not limited to neuronal migration, dendritic growth, synapse maintenance, and long-term plasticity [5]. However, the number of clinical research studies of the role of BDNF polymorphisms in stroke is limited, and the exact influence of BDNF 
polymorphisms underpinning the aspects of stroke severity, recovery, and functional outcome is still unclear [6].

The Val66Met of the BNDF gene, also known as rs6265, is only known to occur in humans and currently remains one of the most studied single-nucleotide polymorphisms of the BDNF gene [4]. In normal functioning, BDNF plays a significant neurological role in the modulation of hippocampal plasticity and hippocampal-dependent memory in humans and animals [4]. On the other hand, Val66Met mutation is associated with a reduction in the hippocampal tissue. Moreover, this mutation is linked hypothetically to several brain diseases, such as memory impairments and neuropsychiatric disorders [7].

The relationship between the language function and variations in the BDNF gene, however, is relatively less prominent. Nevertheless, evidence suggests that BDNF plays a significant role in learning and memory by inducing longterm potentiation (LTP), an essential form of synaptic plasticity [8]. A study by Winter et al. is aimed at investigating the effects of physical exercise and learning performance. They have demonstrated that the peripheral levels of BDNF were increased and sustained more strongly during learning (including language learning) after physical exercise in healthy adults. Here, it proved the role of BDNF as a mediator of exercise-induced learning improvement [9].

The main impetus of the present review is to investigate whether BDNF Val66Met polymorphism is associated with language function in people with poststroke aphasia. We hypothesized that the presence of the BDNF Val66Met polymorphism would affect the language function outcome after stroke.

\section{Material and Methods}

2.1. Search Methodology. Two reviewers conducted a literature search in PubMed and Google Scholar, with the following terms: "brain derived-neurotrophic factor" AND "aphasia" AND "stroke" for articles published from January 2011 to December 2020. The search results were then screened based on the subsequent inclusion and exclusion criteria. Any disagreements were resolved by consulting a third reviewer, if necessary.

2.2. Study Selection. The selected articles must be in English. We considered clinical studies that included BDNF evaluation as part of the main variables among adults with stroke (age 18 years and above). Case reports, review articles, technical reports, and thesis dissertation were excluded, as well as abstract-only publications. Studies that exclusively determined nonlanguage cognitive domains outcomes were also excluded.

2.3. Data Extraction and Recording. The following data were extracted and recorded: (i) details of article (title, author, year of publication, study design, and sample size); (ii) demographic and clinical characteristics of the studied population (mean age, type of stroke, and BDNF genotypes); and (iii) study outcomes (researched variables, interventions, outcome measures of assessment, and their corresponding results). Being a review article, the authors did not request ethical approval as the articles were already published.

\section{Results}

The electronic search resulted in 10 records. After removing duplicates, screening all the titles and abstracts, and accessing full articles of seven studies, a total of 3 articles were selected based on the eligibility criteria. Figure 1 illustrates the PRISM flowchart on the selection process. Table 1 summarizes the characteristics and key findings of the selected articles.

3.1. BDNF Polymorphism Characteristics. All three studies included Val66Met single-nucleotide polymorphism as the studied human BDNF gene [10-12]. de Boer et al. [10] carried out a prospective follow-up study to investigate the effects of the function limiting Val66Met polymorphism of BDNF on the recovery of poststroke aphasia in acute stroke. They divided the affected individuals into two groups based on their BDNF genotype, namely, carriers (with at least 1 Met allele) and noncarriers (absence of Met allele) [10]. A randomized controlled trial by Fridriksson et al. [11] investigated the response of different carriers of BDNF genotypes on behavioural aphasia treatment in acute stroke, while Kristinsson et al. [12] conducted a cross-sectional study to investigate how BDNF genotype may influence functional brain activation in chronic aphasia. Both divided the groupings of polymorphism into typical and atypical-the former is grouped based on having the Val66 Val allele, i.e., BDNF polymorphism in the absence of Met allele. In contrast, the latter has at least one Met allele, either the Val66Met or Met66Met.

3.2. Speech and Language Intervention for Poststroke Aphasia. Only two studies investigated the effect of the intervention on language outcomes $[10,11]$. All participants studied by de Boer et al. [10] received 2 to 5 hours of speech and language therapy (SLT) per week throughout the intake period of 2 years. The primary outcomes were measured using the Amsterdam-Nijmegen Everyday Language Tests (ANELT) and Boston Naming Test (BNT), assessed at baseline and discharge, in which both measures demonstrated improvement over time [10]. All acute stroke participants in the study by Fridriksson et al. [11] received 15 computerized language aphasia treatments, which focused on pictureword matching for 45 minutes, five times per week for three weeks. They were randomized to receive either $1 \mathrm{~mA}$ of anodal tDCS (transcranial direct stimulation current) or sham tDCS to the left temporoparietal region for the first 20 minutes of each session [11]. The therapeutic response to tDCS was assessed using the Philadelphia Naming Test (PNT), "Naming 80" test, and Western Aphasia Battery (WAB) test at one week, four weeks, and 24 weeks posttreatment [11].

3.3. Influences of BDNF Polymorphism on Poststroke Aphasia Recovery. Even though there were improvements in both ANELT and BNT, de Boer et al. [10] failed to demonstrate significant differences in both performances between the two groups at discharge, despite a large discrepancy in the 


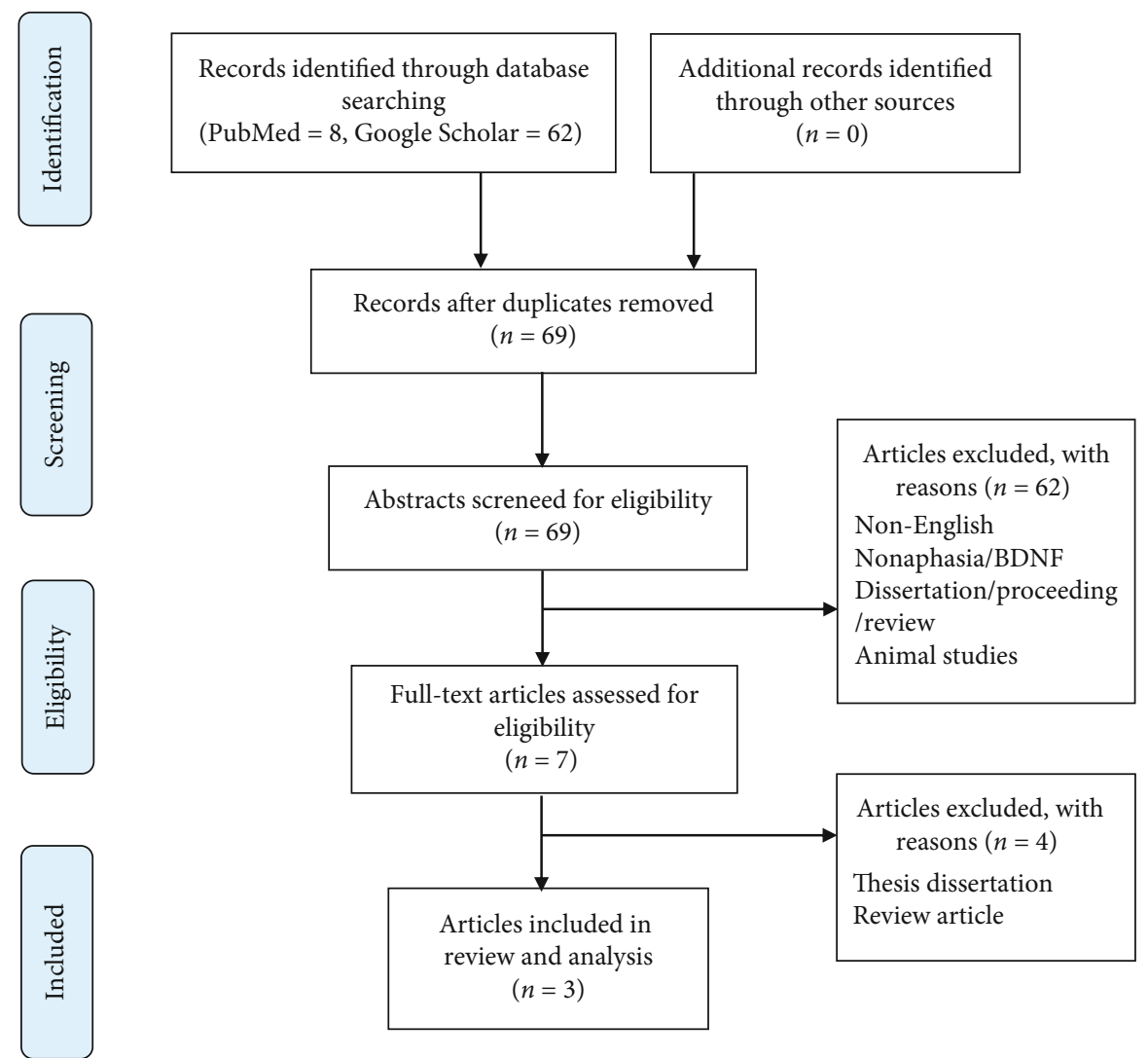

FIGURE 1: PRISM flowchart of the article selection process.

baseline scores and the improvement scores across both groups. The study did not control confounding variables, including stroke severity, conditions for discharge, and social factors in this study [10]. The differences in the improvements of both the ANELT and BNT between both groups were not statistically significant [10]. The findings from this preliminary study suggested that there is insufficient evidence to conclude that BDNF polymorphism plays a role in poststroke aphasia recovery.

Based on the study by Fridriksson et al. [11], the baseline aphasia quotient (AQ) scores from revised Western Aphasia Battery (WAB) demonstrated that atypical BDNF genotype carriers had a more severe aphasia presentation than typical BDNF genotype carriers [11]. This result was consistent with the presumption that the atypical BDNF genotype leads to lower levels of BDNF secretion during activity [11]. Moreover, typical BDNF genotype patients exhibited improvement in naming for both A-tDCS and sham tDCS interventions [11]. Interestingly, contrary to the results from de Boer et al.'s study [10], Fridriksson et al. [11] demonstrated that the BDNF Met allele genotype has an impact on language performance and improvement in stroke. Furthermore, the latter showed that Met allele carriers of the BDNF gene produced a more unsatisfactory response to aphasia treatment than $\mathrm{Val} 66 \mathrm{Val}$ and other typical genotype carriers of BDNF, regardless of the language therapy delivered [11]. In addition, Fridriksson et al. found no differences for other factors such as semantic processing, executive func- tion, stroke severity, age, lesion size, education, or time poststroke between both groups [11].

Kristinsson et al. [12] used functional magnetic resonance imaging (MRI) for visualizing the cortical activation and WAB for measuring language impairment in two groups of participants with chronic stroke based on typical or atypical BNDF polymorphism carrier status [12]. First, the naming-related activation lesion contrast maps showed a relatively lesser activation present in the right hemisphere of the atypical group than the typical group [12]. Following this, they further quantified the MRI finding by obtaining the number of voxels present in predetermined regions of functional naming-related activated regions for each group of participants at the whole-brain level and both the left and right hemispheres, respectively and separately [12]. The typical genotype group demonstrated a higher number of activated voxels than the atypical group at both the wholebrain and right hemispheres [12].

In addition, participants in the atypical BDNF group had an overall greater aphasia severity on the revisedWAB-AQ than that of typical BDNF carriers of chronic stroke [12]. The findings of this study suggest that cortical brain activation is potentially mediated by BDNF genotypes, with reduced cortical activation of Met allele carriers [12]. There were no significant differences between both groups for baseline stroke severity, baseline aphasia severity, and executive functioning [12]. Age, racial distribution, education, lesion size, amount of exercise, or stroke 


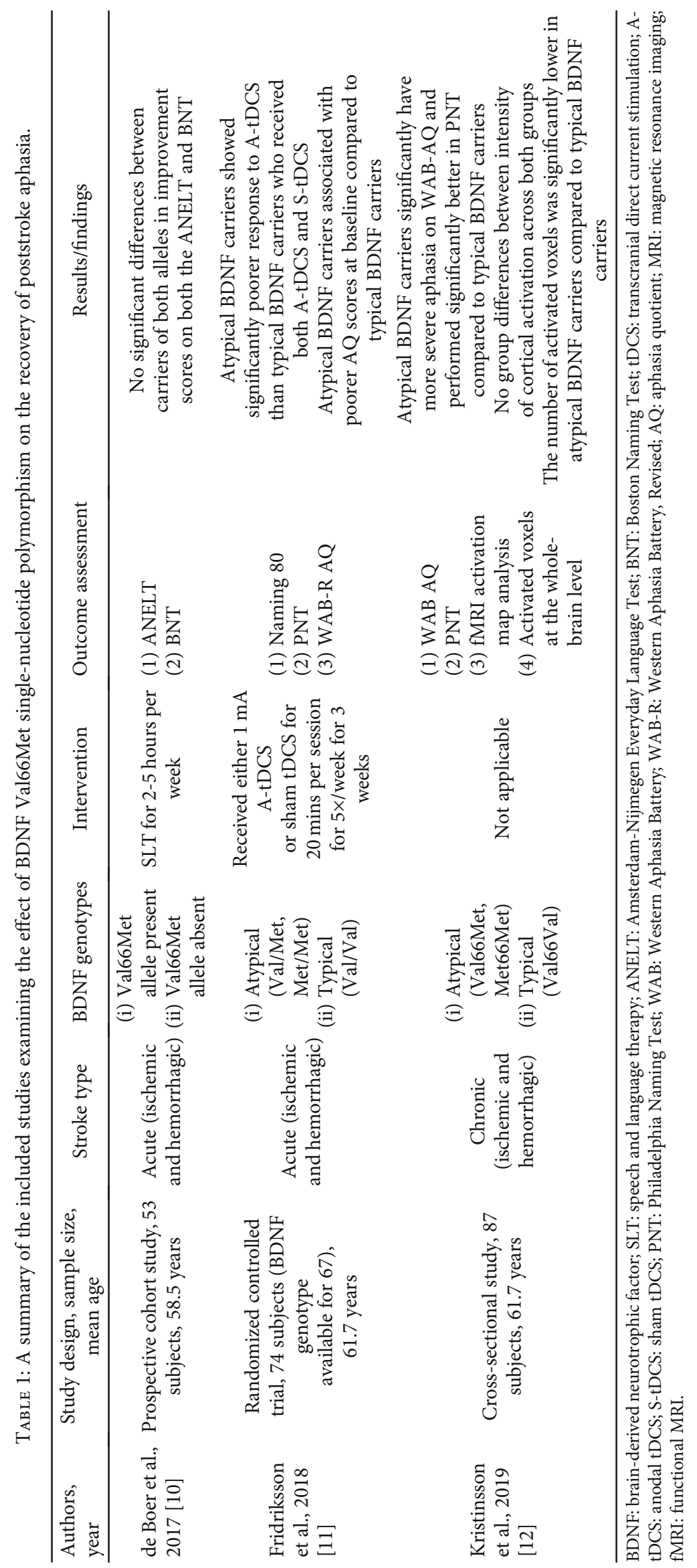


severity differed between the two groups but reached nonsignificant levels [12].

\section{Discussion}

The majority of the results are primarily in line with established evidence when evaluating the impacts of BDNF polymorphism on poststroke outcomes $[3,6]$. Typically, carriers of the Met allele of BDNF presented with poorer long-term functional outcomes after stroke $[13,14]$.

In addition, specific polymorphisms in the human BDNF gene are often linked to greater cognitive performance, including learning and memory, attention, and executive functions $[4,5]$. Thus, it would be reasonable to assume that certain genetic variations in the BDNF gene are affiliated with language production and comprehension.

From the literature search performed, only three studies yielded the investigation of the correlation between language impairment or aphasia in stroke and BDNF genotypes, highly suggesting that knowledge in this topic of interest is relatively new and limited. Based on these findings, the Val66Met polymorphism of BDNF is linked with more severe aphasia at baseline $[11,12]$, poorer improvement in language improvement with time [11], and reduced cortical activation [12]. However, the exact role of BDNF polymorphisms in language performance and recovery in stroke may require further investigation.

The findings of de Boer et al. [10] demonstrated that the BDNF genotype is not specific to language performance and improvement, in contrast to the results of the other selected studies, which showed that BDNF genotypes are involved in the language outcome in stroke $[13,14]$. Several possible explanations can be stipulated for such discrepancy. First, the study by de Boer et al. [10] received a relatively higher frequency of SLT compared to the intervention in [11]. In contrast, the study by Kristinsson et al. [12] did not account for the presence of SLT. Secondly, there were different standardized aphasia tests to assess aphasia: Dutch [10] and English $[11,12]$. Here, the potential effects of bilingualism or multilingualism might require further investigation. Evidence suggests that bilingualism may be protective for adults with aphasia, possibly contributing to cognitive reserve in adults with aphasia [15]. Another consideration in these three prospective studies is the distinction between language recovery and language learning processes during stroke rehabilitation [10]. The significant variation in the improvement scores on the ANT and BNT further complicated the ability to detect significant differences between groups. Lastly, apraxia, which may affect the study results, was not excluded from the study [10].

Although the Val66Met allele of BDNF is associated with poorer language performance after tDCS intervention in poststroke aphasia [11], Marangolo et al. [16] demonstrated that the tDCS does not significantly alter the levels of BDNF on chronic aphasia patients. Thus, despite observing improvement in the scores of language performance, BDNF is not solely responsible for such improvement in language recovery after stroke. Fridriksson et al. [11] have hypothe- sized that this finding could be due to anodal tDCS (A-tDCS) dependence on baseline levels of BDNF secretion.

Contrary to the current theory that the Met allele of BDNF is linked with the defective intracellular secretion of BDNF, Lang et al. [17] have demonstrated that the Val66Met polymorphism of BDNF is associated with increased BDNF serum concentrations instead in healthy subjects. Furthermore, Lang et al. [17] postulated that the Met allele does not affect the constitutive secretion of BDNF but rather decreases the amount of activity-dependent BDNF secretion. Interestingly, Gajewski et al. [18] have shown that healthy elderly carriers of the Met allele of BDNF Val66Met outperformed homozygote ( $\mathrm{Val} / \mathrm{Val}$ ) carriers of BDNF in task switching based on a cue-based and memory-based task. Their findings hypothesized that the Met allele contributes to more efficient cognitive processes under particular circumstances in healthy elderly subjects [18].

In addition, a study by Jasińska et al. [19] investigating the effects of BDNF Val66Met polymorphism on reading ability in children has shown that Met allele carriers of the BDNF gene experienced greater neural activation in the reading-related regions of the brain during a reading task. The performance of the Met allele carriers suggests that the BDNF polymorphism may be associated with phonological working memory, which is crucial in reading ability [19]. Moreover, Freundlieb et al. [20] have failed to find an association between BDNF Val66Met polymorphism and implicit short-term associative language learning paradigms in healthy adults.

Despite appreciating many established associations between variations of BDNF gene in stroke [3] with cognitive impairment and psychiatric disorders [7], the pondering question is whether BDNF can be considered a "disease susceptibility gene". For stroke, BDNF Val66Met polymorphism is associated with long-term functional outcomes, with Met allele carriers exhibiting poorer modified Rankin scale scores $[14,15]$. Nevertheless, there were weak associations between the BNDF gene and psychiatric conditions such as bipolar disorder [21]. Petryshen et al. [21] suggested that the variability in BDNF associations with psychiatric disorders could be attributed to the differences in population genetic structure. Hence, the diversity of BDNF polymorphism among worldwide populations would provide important implications for the implementation of further studies on poststroke aphasia. Furthermore, Kim et al. [13] have suggested that ethnic variability in the frequency of distribution of alleles may affect the positive findings to detect associations between BDNF genotypes and stroke outcomes.

Therefore, the BDNF gene may show a significant association with aphasia recovery after stroke, with the Met allele of the gene linked to poorer language recovery. In conclusion, some evidence suggests that polymorphism in the BDNF gene may modulate language recovery in poststroke aphasia. However, future research would be required to understand better the relationship between BDNF genetic variations and poststroke aphasia.

4.1. Study Limitations. The current review has several limitations. Firstly, there are a relatively limited number of original 
articles on the topic of BDNF polymorphism and poststroke aphasia-related outcomes, most prominently in the scope of BDNF genotypes. Hence, making strong inferences from a limited set of results concerning a topic as complex as the role of genetic polymorphism in aphasia would be challenging. In addition, there is variability in the parameters, interventions, and outcome measures utilized by the researchers. Finally, certain uncontrolled variables such as time of onset after stroke, type of aphasia, and presence and intensity of SLT have rendered the study populations as heterogeneous groups, which may have led to insufficient evidence for further statistical meta-analysis. These limitations justify future studies to explore the association between BDNF polymorphism and poststroke aphasia especially considering the emergence of neuromodulation therapy that promotes language improvement. In addition, establishing a more objective connection between these genotyping and the recovery of aphasia after stroke would enhance a better patients' selection for better utilization of resources.

\section{Conclusion}

There is some evidence suggesting that the Met allele of BDNF is associated with poorer language outcome in poststroke patients, in both acute and chronic stages. Further works are warranted to investigate this association to explore future treatments and strategies, which may produce therapeutic effects more efficiently for stroke patients. Employing an assumption that BDNF Val66Met polymorphism influences the severity and recovery of aphasia, identifying specific alleles of BDNF as a predictor for aphasia severity and recovery may be the next step targeting selective therapeutic strategies in stroke patients. However, our current understanding of the influence of specific genes in aphasia recovery is still relatively limited. Based on the findings of the selected articles, it seems that a correlation between BDNF polymorphism and aphasia recovery exists, although the exact mechanisms underpinning this effect are still unclear. Advancement in the study of the genetic influencers of aphasia may provide more efficient therapies for people with aphasia, therefore potentially improving the current prognosis of aphasia.

\section{Data Availability}

In view of the nature of the article, the authors do not own the data and all sources are cited appropriately.

\section{Conflicts of Interest}

The authors declare that there is no conflict of interest regarding the publication of this article.

\section{Acknowledgments}

This work is supported by a research grant from Universiti Malaysia Sabah (grant number: GUG4581/2020).

\section{References}

[1] G. A. Donnan, M. Fisher, M. Macleod, and S. M. Davis, "Stroke," The Lancet, vol. 371, no. 9624, pp. 1612-1623, 2008.

[2] M. Kotila, O. Waltimo, M. L. Niemi, R. I. Laaksonen, and M. A. Lempinen, "The profile of recovery from stroke and factors influencing outcome," Stroke, vol. 15, no. 6, pp. 10391044, 1984.

[3] M. Balkaya and S. Cho, "Genetics of stroke recovery: BDNF val66met polymorphism in stroke recovery and its interaction with aging," Neurobiology of Disease, vol. 126, pp. 36-46, 2019.

[4] M. F. Egan, M. Kojima, J. H. Callicott et al., "The BDNF val66met polymorphism affects activity-dependent secretion of BDNF and human memory and hippocampal function," Cell, vol. 112, no. 2, pp. 257-269, 2003.

[5] A. K. McAllister, "BDNF," Current Biology, vol. 12, no. 9, article R310, 2002.

[6] T. M. Stanne, A. Tjärnlund-Wolf, S. Olsson, K. Jood, C. Blomstrand, and C. Jern, "Genetic variation at the BDNF locus: evidence for association with long-term outcome after ischemic stroke," PLoS One, vol. 9, no. 12, article e114156, 2014.

[7] K. G. Bath and F. S. Lee, "Variant BDNF (Val66Met) impact on brain structure and function," Cognitive, Affective, \& Behavioral Neuroscience, vol. 6, no. 1, pp. 79-85, 2006.

[8] C. Cunha, R. Brambilla, and K. L. Thomas, "A simple role for BDNF in learning and memory?," Frontiers in Molecular Neuroscience, vol. 3, p. 1, 2010.

[9] B. Winter, C. Breitenstein, F. C. Mooren et al., "High impact running improves learning," Neurobiology of Learning and Memory, vol. 87, no. 4, pp. 597-609, 2007.

[10] R. G. de Boer, K. Spielmann, M. H. Heijenbrok-Kal, R. van der Vliet, G. M. Ribbers, and W. M. van de Sandt-Koenderman, "The role of the BDNF Val66Met polymorphism in recovery of aphasia after stroke," Neurorehabilitation and Neural Repair, vol. 31, no. 9, pp. 851-857, 2017.

[11] J. Fridriksson, J. Elm, B. C. Stark et al., "BDNF genotype and tDCS interaction in aphasia treatment," Brain Stimulation, vol. 11, no. 6, pp. 1276-1281, 2018.

[12] S. Kristinsson, G. Yourganov, F. Xiao et al., "Brain-derived neurotrophic factor genotype-specific differences in cortical activation in chronic aphasia," Journal of Speech, Language, and Hearing Research, vol. 62, no. 11, pp. 3923-3936, 2019.

[13] J. M. Kim, R. Stewart, M. S. Park et al., "Associations of BDNF genotype and promoter methylation with acute and long-term stroke outcomes in an East Asian cohort," PLoS One, vol. 7, no. 12, article e51280, 2012.

[14] J. Zhao, H. Wu, L. Zheng, Y. Weng, and Y. Mo, "Brain-derived neurotrophic factor G196A polymorphism predicts 90-day outcome of ischemic stroke in Chinese: a novel finding," Brain Research, vol. 1537, pp. 312-318, 2013.

[15] M. Dekhtyar, S. Kiran, and T. Gray, "Is bilingualism protective for adults with aphasia?," Neuropsychologia, vol. 139, p. 107355, 2020.

[16] P. Marangolo, V. Fiori, F. Gelfo et al., "Bihemispheric tDCS enhances language recovery but does not alter BDNF levels in chronic aphasic patients," Restorative Neurology and Neuroscience, vol. 32, no. 2, pp. 367-379, 2014.

[17] U. E. Lang, R. Hellweg, T. Sander, and J. Gallinat, “The Met allele of the BDNF Val66Met polymorphism is associated with 
increased BDNF serum concentrations," Molecular Psychiatry, vol. 14, no. 2, pp. 120-122, 2009.

[18] P. D. Gajewski, J. G. Hengstler, K. Golka, M. Falkenstein, and C. Beste, "The Met-allele of the BDNF Val66Met polymorphism enhances task switching in elderly," Neurobiology of Aging, vol. 32, no. 12, pp. 2327.e7-2327.e19, 2011.

[19] K. K. Jasińska, P. J. Molfese, S. A. Kornilov et al., "The BDNF Val66Met polymorphism influences reading ability and patterns of neural activation in children," PLoS One, vol. 11, no. 8, article e0157449, 2016.

[20] N. Freundlieb, S. Philipp, S. A. Schneider et al., "No association of the BDNF Val66met polymorphism with implicit associative vocabulary and motor learning," PLoS One, vol. 7, no. 11, article e48327, 2012.

[21] T. L. Petryshen, P. C. Sabeti, K. A. Aldinger et al., "Population genetic study of the brain-derived neurotrophic factor $(B D N F)$ gene," Molecular Psychiatry, vol. 15, no. 8, pp. 810-815, 2010. 